\title{
Methane and the Spectra of T Dwarfs
}

\author{
Derek Homeier and Peter H. Hauschildt \\ Department of Physics 6 Astronomy and Center for Simulational \\ Physics, University of Georgia, Athens, GA 30602-2451 \\ France Allard \\ CRAL, Ecole Normale Supérieure, Lyon, France
}

\begin{abstract}
We have updated our PHOENIX model atmospheres and theoretical spectra for ultracool dwarfs with new opacity data for methane based on line strength predictions with the STDS software. By extending the line list to rotational levels of $J=40$ we can significantly improve the shape of the near-IR absorption features of $\mathrm{CH}_{4}$, and in addition find an enhanced blanketing effect, resulting in up to $50 \%$ more flux emerging in the $J$ band than seen in previous models, which may thus contribute to the brightening in $J$ and blue IR colors observed in T dwarfs.
\end{abstract}

\section{Introduction}

Absorption bands of $\mathrm{H}_{2} \mathrm{O}$ and $\mathrm{CH}_{4}$ dominate the IR spectra of dwarfs cooler than $\sim 1400 \mathrm{~K}$ and are a backbone of the $\mathrm{T}$ dwarf classification schemes defined by Geballe et al. (2002) and Burgasser et al. (2002). Accurate and complete opacity data for these molecules are thus essential for modelling the atmospheres of $\mathrm{T}$ dwarfs. We have updated the PHOENIX model atmosphere code with a new methane list based on theoretical calculations with the Spherical Top Data System (STDS) (Wenger \& Champion 1998). Compared to our AMES-Cond models (Allard et al. 2001), with $\sim 37000 \mathrm{CH}_{4}$ lines from the HITRAN and GEISA databases, this has added $1.2 \cdot 10^{7}$ lines (Homeier et al. 2002).

\section{Theoretical Spectra}

We have computed opacity sampling spectra in the limiting case of fully settled dust clouds as described in Allard et al. (2001). The main difference in the line lists is a much larger set of faint lines, mostly due to a coverage in rotational quantum number extending up to $J=40$, as compared to typically $J \leq 10$ in the HITRAN and GEISA data. We discuss the changes in these models on the case of the T 4.5 dwarf SDSS $0207+00$ observed by Geballe et al. (2002). As can be seen in the absorption feature around $2.2 \mu \mathrm{m}$, rotational levels up to about $J=30$ need to be included to reproduce the band shape over its full width.

Less evident in the spectra is the dense background of faint lines at higher $J$, which effectively closes any windows of small opacity throughout all of the $H$ 

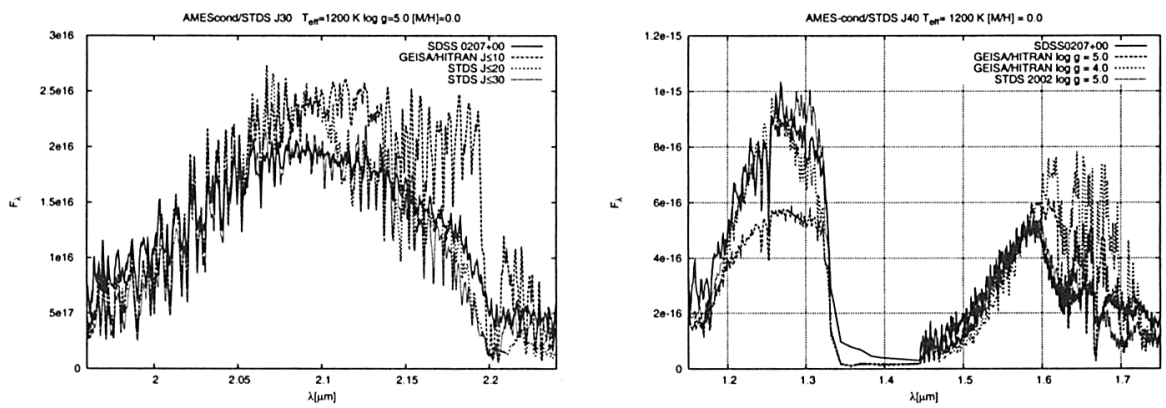

Figure 1. AMES-Cond models and new STDS models compared to an observed T dwarf spectrum: $K$ band region with STDS lines up to $J$ of 20 and 30, respectively (left); $J$ and $H$ band region with $\log g=5.0$ models and a $\log g=4.0$ AMES-Cond model for comparison (right).

and $K$ bands. This results in a stronger redistribution of flux towards shorter wavelengths, and thus a brightening of the $J$ band of about $0 .{ }^{\mathrm{m}} 5$ compared to an AMES-Cond model with the same parameters. We note that the colors can be "fixed" with lower $\log g$ models in the AMES-Cond grid, but at the cost of an even worse spectral fit in the $H$ and $K$ bands.

\section{Conclusions}

We could significantly improve our synthetic IR spectra for $\mathrm{T}$ dwarfs with the new STDS line list for methane. In addition, the new models produce a shift of emergent flux towards the $J$ band due to stronger blanketing, reproducing well the blue IR colors of $\mathrm{T}$ dwarfs. To clarify the exact role of the onset of $\mathrm{CH}_{4}$ in the rapid brightening in $J$ observed at the L-T transition, a new set of models will be necessary that also provides a consistent treatment of the settling of the cloud layer below the photosphere expected to occur in about the same temperature range (Allard et al., these proceedings).

Acknowledgments. We thank D. Alexander, V. Boudon, J.-P. Champion and U. Jørgensen for helpful discussions and S. Leggett for access to observational data. This work is supported by NFS grant N-Stars RR185-258.

\section{References}

Allard, F., Hauschildt, P. H., Alexander, D. R. et al. 2001, ApJ 556, 357 Burgasser, A. J., Kirkpatrick, J. D., Brown, M. E. et al. 2002, ApJ 564, 421 Geballe, T. R., Knapp, G. R. and Leggett, S. K. et al. 2002, ApJ 564, 466 Homeier, D., Hauschildt, P. H. and Allard, F. 2002, in: Stellar Atmosphere Modeling, eds. K. Werner, I. Hubeny \& D. Mihalas, PASP, in press

Wenger, C. \& Champion, J. P. 1998, JQSRT 59, 471 